\title{
Nitrogen permease regulator-like 2 enhances sensitivity to oxaliplatin in colon cancer cells
}

\author{
MING-NA LIU, AI-YUN LIU, YA-JU DU, FENG-HUA PEI, XIN-HONG WANG, \\ JING CHEN, DAN LIU and BING-RONG LIU
}

\begin{abstract}
Department of Gastroenterology, The Second Affiliated Hospital of Harbin Medical University, Harbin, Heilongjiang 150080, P.R. China
\end{abstract}

Received June 18, 2014; Accepted February 17, 2015

DOI: $10.3892 / \mathrm{mmr} .2015 .3495$

\begin{abstract}
Colorectal cancer (CRC) is the third most common cancer worldwide. Chemotherapeutic compounds used for the treatment of CRC include oxaliplatin (L-OHP). While L-OHP improves CRC survival, certain patients are resistant. The nitrogen permease regulator like-2 (NPRL2) gene is a candidate tumor suppressor gene that resides in a 120-kb homozygous deletion region on chromosome 3p21.3. In the present study, it was demonstrated that NPRL2 overexpression increases the sensitivity of HCT116 cells to L-OHP. The IC50 of L-OHP was decreased in cells transduced with NPRL2 compared with negative control (NC) cells and the effect of NPRL2 on L-OHP sensitivity was time dependent. Following NPRL2 transduction in HCT116 cells, the cell cycle was arrested in the G1 phase and a partial decrease in the S phase population was observed. Flow cytometric analysis revealed that NPRL2 transduction and L-OHP treatment increased apoptosis compared with NC cells. The mechanism through which NPRL2 overexpression enhances L-OHP sensitivity involves downregulation of the functions of the phosphatidylinositol 3-kinase/Akt/mammalian target of rapamycin network. Furthermore, L-OHP upregulated caspase-3 and caspase-9 to promote apoptosis in NPRL2-overexpressing cells compared with cells that were transduced with NPRL2 or treated with $\mathrm{L}-\mathrm{OHP}$ and NC cells $(\mathrm{P}<0.01)$. NPRL2 overexpression led to the downregulation of $\mathrm{CD} 24$, which could significantly reduce tumor invasiveness and decrease the metastatic capacity of HCT116 cells. These mechanisms are likely active in other types of cancer and may be exploited for the development of novel cancer therapies.
\end{abstract}

Correspondence to: Professor Bing-Rong Liu, Department of Gastroenterology, The Second Affiliated Hospital of Harbin Medical University, 148 Baojian Road, Harbin, Heilongjiang 150080, P.R. China

E-mail: liubingrong@medmail.com.cn

Key words: nitrogen permease regulator-like 2, colorectal cancer, phosphatidylinositol 3-kinase/Akt/mammalian target of rapamycin, oxaliplatin

\section{Introduction}

Colorectal cancer (CRC) ranks third in worldwide cancer incidence and second in mortality, with $\sim 1.2$ million new patients diagnosed per year (1). In China, CRC has become the third leading cause of mortality due to tumor disease. Genetic and environmental risk factors, including lifestyle and nutrition modulate colon cancer development (2). Chemotherapeutic compounds used for the treatment of CRC include oxaliplatin (L-OHP), 5-flurouracil and irinotecan (3). While chemotherapy can improve the survival rate of CRC patients, resistance emerges in certain patients, with a typical survival of $<6$ months once resistance is identified. The detection of genes associated with CRC may facilitate the development of targeted therapies to improve CRC prognosis, reduce resistance and adverse events and establish individualized treatment programs for CRC.

The nitrogen permease regulator like-2 (NPRL2) gene is a candidate tumor suppressor gene identified in the 3 p21.3 region. Genomic abnormalities have been identified in this region in various types of human cancer $(4,5)$. Certain studies suggest that the NPRL2 gene may be a tumor suppressor and that its inactivation may promote tumorigenesis (4-6). The NPRL2 gene is composed of 11 exons and encodes a 380 amino acid protein. Multiple spliced isoforms of NPRL2 are expressed in different tissue types. However, the mechanism by which NPRL2 mediates tumor suppression remains to be elucidated. Previous studies have suggested that NPRL2 is involved in DNA mismatch repair, cell cycle checkpoint signaling and the regulation of apoptosis $(4,6)$. In certain tumor cell lines, overexpression of NPRL2 induces apoptosis and inhibits proliferation (7).

NPRL2 has been demonstrated to increase susceptibility to anticancer drugs and apoptosis $(7,8)$. Previous studies have reported that NPRL2 is a potential biomarker for predicting response to cisplatin, the prognosis of patients with lung cancer and other types of cancer and as a molecular therapeutic agent for enhancing and resensitizing the response of nonresponders to cisplatin treatment $(7,8)$. However, how NPRL2 suppresses tumor proliferation and whether NPRL2 can affect the sensitivity of cells to chemotherapy remains to be elucidated. In the present study, the colon cancer cell line HCT116 was used to observe the effects of the NPRL2 signaling pathway on 
apoptosis induced by the chemotherapeutic drug L-OHP to further elucidate the role of the NPRL2 signaling pathway in increased L-OHP sensitivity in these cells as part of the search for an effective treatment for CRC.

\section{Materials and methods}

Cell culture. The colon cancer cell line HCT116 was purchased from the Chinese Academy of Sciences (Shanghai, China). The cells were cultured in RPMI-1640 medium supplemented with $10 \%$ fetal bovine serum (HyClone Laboratories, Inc., Logan, UT, USA) and $1 \%$ penicillin/streptomycin (Beyotime Institute of Biotechnology, Haimen, China) in a humidified atmosphere of $5 \% \mathrm{CO}_{2}$ at $37^{\circ} \mathrm{C}$. Cells were passaged every 2-3 days through digestion with $0.25 \%$ trypsin. Logarithmically growing cells were prepared.

Transductions and assay. The full length human NPRL2 gene (GenBank serial number: NM_006545) was purchased from Shanghai Genechem Co., Ltd. (Shanghai, China) as a fusion with enhanced green fluorescence protein (eGFP) in the GV208 vector. The lentiviral vector system consisted of GV208 and the pHelper 1.0 and pHelper 2.0 packaging vectors. The three vectors were cotransfected into $293 \mathrm{~T}$ cells in serum-free medium using Lipofectamine 2000 (Invitrogen Life Technologies, Carlsbad, CA, USA). The medium was changed to complete medium after $8 \mathrm{~h}$ of incubation. High-titer recombinant lentiviruses encoding NPRL2 were harvested $48 \mathrm{~h}$ after transfection. HCT116 cells in the log phase were seeded at $5 \times 10^{5}$ cells/well in 96-well plates and transduced with NPRL2-GFP or GFP lentiviruses in serum-free medium. Polybrene was added to improve the transduction efficiency. After $8 \mathrm{~h}$, the medium was changed to complete medium. At $72 \mathrm{~h}$ after transduction, GFP expression was examined by fluorescence microscopy (Nikon TE2000; Nikon Corporation, Tokyo, Japan) and a luciferase assay was performed in HCT116 cells. The protein expression levels were analyzed $72 \mathrm{~h}$ after transduction. All experiments were performed in triplicate and representative results are reported.

Cell viability assay. Non-transduced and transduced cells were dispersed and seeded at $5 \times 10^{3}$ cells/well in 96-well microplates. After 24 h, freshly prepared L-OHP (Sanofi-Synthelabo, Paris, France) was used to determine the optimal concentration and time course of the HCT116 cell response to L-OHP. Cell viability was assessed using a cell counting kit-8 assay (Dojindo Laboratories, Kumamoto, Japan) following various concentrations of drug treatment $(2.5,5,10,20$ and $40 \mu \mathrm{g} / \mathrm{ml} \mathrm{L-OHP})$ or duration in culture (24, 48 and $72 \mathrm{~h})$. The absorbance value at $450 \mathrm{~nm}$ was read using a microplate reader (Multiskan MK3; Thermo Fisher Scientific Inc., Rockford, IL, USA). At least three independent experiments were performed in quadruplicate.

Flow cytometric analysis of the cell cycle and apoptosis. Cells transduced with NPRL2 were treated with $10 \mu \mathrm{g} / \mathrm{ml}$ $\mathrm{L}-\mathrm{OHP}$ for $48 \mathrm{~h}$ and harvested. Following trypsinization, the cells were washed with phosphate-buffered saline (PBS; Beyotime Institute of Biotechnology) and subsequently fixed in $85 \%$ ethanol. Following fixation, the cells were washed with
PBS/1\% fetal calf serum (FCS; HyClone Laboratories, Inc.), resuspended in $\mathrm{PBS} / 1 \%$ FCS containing $10 \mu \mathrm{g} / \mathrm{ml}$ propidium iodide (PI) and $250 \mu \mathrm{g} / \mathrm{ml}$ RNase A (Multisciences Biotech Co., Ltd., Hangzhou, China) and incubated for $30 \mathrm{~min}$ at $37^{\circ} \mathrm{C}$. Apoptosis was evaluated using flow cytometry with Annexin V-fluorescein isothiocyanate and PI (KeyGEN Biotech Co., Ltd., Nanjing, China) staining. Following gating on the $\mathrm{CD}_{24}{ }^{+}$subpopulation, the cells were analyzed for positive Annexin V staining. Positive rates of CD24+ apoptosis were detected using a FACScan flow cytometer (Becton Dickinson, Franklin Lakes, NJ, USA) following the addition of CD24 monoclonal antibodies (Ebioscience, San Diego, CA, USA).

Western blot analysis. Cellular protein extracts were separated by electrophoresis in a 12 or $8 \%$ SDS-polyacrylamide gel (Beyotime Institute of Biotechnology) and electrophoretically transferred onto a polyvinylidene fluoride membrane (Millipore, Bedford, MA, USA). The membranes were blocked overnight with $5 \%$ non-fat dried milk and incubated overnight at $4^{\circ} \mathrm{C}$ with the following antibodies: Rabbit monoclonal anti-GAPDH (cat. no. 2118; 1:1,500), rabbit monoclonal anti-pyruvate dehydrogenase kinase, isozyme 1 (PDK1) (cat. no. 13037; 1:1,000), mouse monoclonal anti-p-Akt (cat. no. 4051; 1:1,000), rabbit monoclonal anti-mammalian target of rapamycin (mTOR)(p) (cat. no. 5536; 1:1,000), rabbit polyclonal anti-p70S6K(P) (cat. no. 9209; 1:1,000) and rabbit monoclonal anti-4E-binding protein 1 (4E-BP1) (cat. no. 9456; 1:1,000) purchased from Cell Signaling Technology, Inc. (Danvers, MA, USA) or mouse monoclonal anti-NPRL2 (cat. no. sc-376986; 1:1,000), rabbit polyclonal anti-phosphatidylinositol 3-kinase (PI3K)(p) (cat. no. sc-134986; 1:1,000), rabbit polyclonal anti-caspase-3 (cat. no. sc-7148; 1:1,000) and mouse monoclonal anti-caspase-9 (cat. no. sc-56073; 1:2,000) obtained from Santa Cruz Biotechnology, Inc. (Santa Cruz, CA, USA). Following washing with Tris-buffered saline and Tween 20, the membranes were incubated with a horseradish peroxidase-linked goat anti-rabbit (cat. no. A0208; 1:1,000) or goat anti-mouse (cat. no. A0216; 1:1,000) immunoglobulin G secondary antibodies (Beyotime Institute of Biotechnology). The proteins were visualized by enhanced chemiluminescence using an integrated automatic chemiluminescent imaging and analysis system (Sage Creation Science Co., Ltd., Beijing, China).

Statistical analysis. All experimental data are presented as the mean \pm standard error of the mean. Differences between samples were analyzed using the two-tailed Student's t-test. $\mathrm{P}<0.05$ was considered to indicate a statistically significant difference.

\section{Results}

Lentiviral transduction of NPRL2. Transduction efficiency was evaluated $72 \mathrm{~h}$ after NPRL2 transduction. eGFP was expressed in cells following lentiviral transduction at different multiplicities of infection (MOIs). The transduction efficiency (average proportion of GFP-expressing cells compared with the total cell count) was $>70 \%$ at an MOI of $10 \quad(\mathrm{P}<0.05$; Fig. 1A-D). Protein expression levels were analyzed at $72 \mathrm{~h}$ post-transduction. NPRL2 expression was higher in the 
A

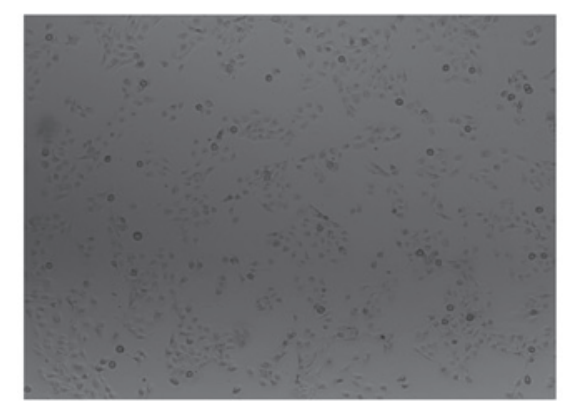

C

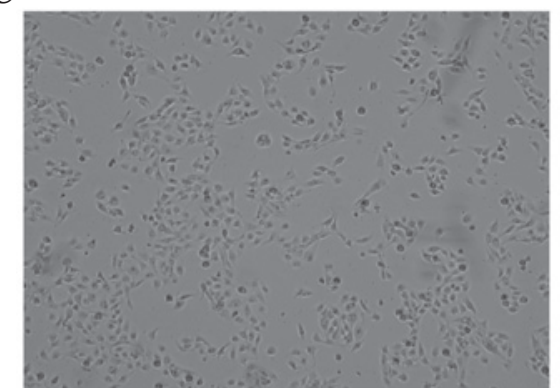

$\mathbf{E}$

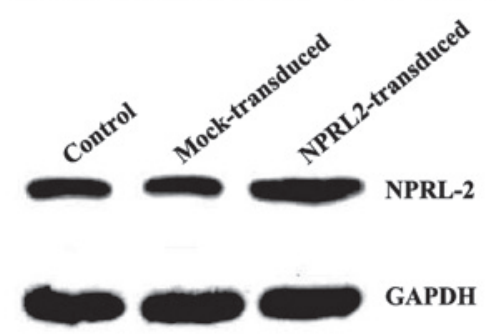

B

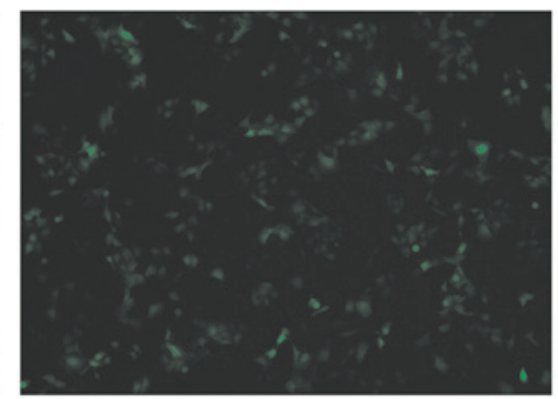

D
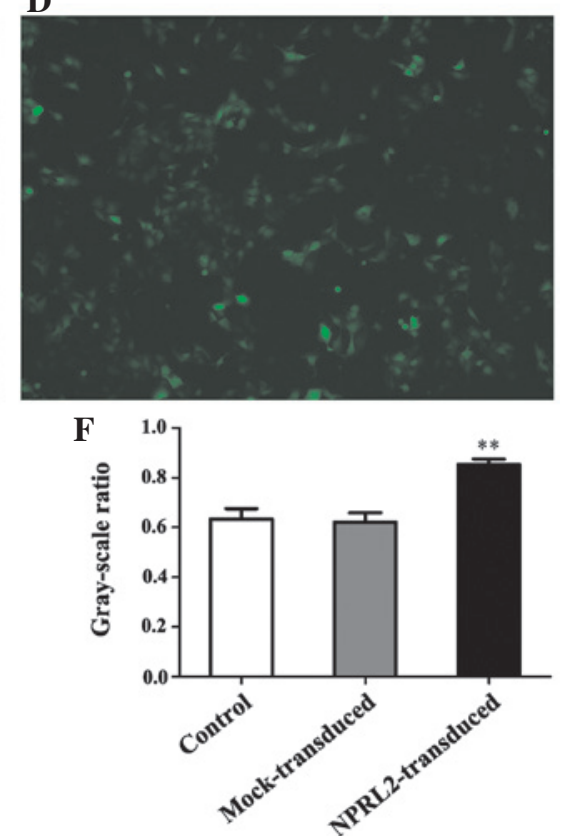

Figure 1. Monitoring of the transduction efficiency of HCT116 cells by light and fluorescence microscopy. (A and B) Mock-transduced HCT116 cells and (C and D) NPRL2-transduced HCT116 cells. (E and F) NPRL2 protein expression levels were also analyzed. ${ }^{* *} \mathrm{P}<0.01$, as compared with the mock-transduced control cells. NPRL2, nitrogen permease regulator-like 2.

$\mathbf{A}$

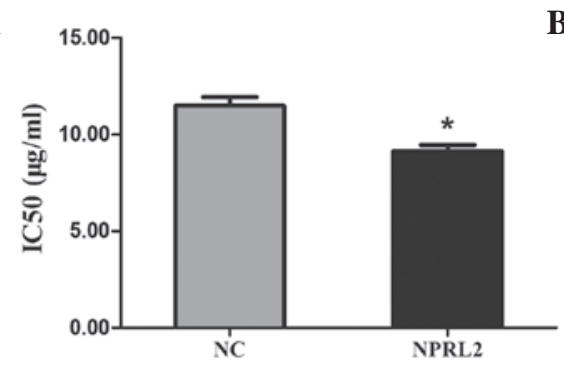

B

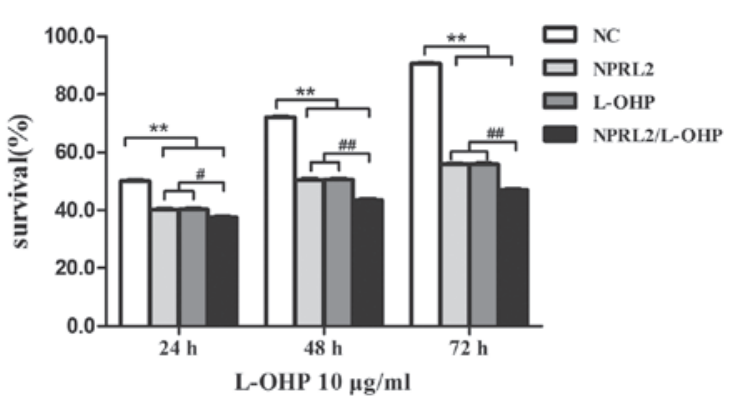

Figure 2. NPRL2 increases the sensitivity of HCT116 cells to L-OHP. (A) The IC50 of L-OHP was lower in cells transduced with NPRL2 than in NC cells. ${ }^{*} \mathrm{P}<0.05$, as compared with the NC cells. (B) Cell survival was assayed following NPRL2 transduction and treatment with $10 \mu \mathrm{g} / \mathrm{ml} \mathrm{L}-\mathrm{OHP}$ for an additional 24, 48 and 72 h. ${ }^{\# P}<0.05$, compared with NPRL2-transduced cells, cells treated with L-OHP for 24 h; ${ }^{\# P} \mathrm{P}<0.01$, compared with NPRL2-transduced cells, cells treated with L-OHP for 48 and $72 \mathrm{~h} ;{ }^{* *} \mathrm{P}<0.01$, compared with NPRL2-transduced cells, cells treated with L-OHP, NPRL2-transduced cells treated with L-OHP for 24, 48 and $72 \mathrm{~h}$. NC, negative control; NPRL2, nitrogen permease regulator-like 2; L-OHP, oxaliplatin.

transduced cells than in the negative control (NC) and mock cells $(\mathrm{P}<0.01$; Fig. $1 \mathrm{E}$ and $\mathrm{F})$.

NPRL2 overexpression increases the L-OHP sensitivity of HCT116 cells. To investigate the role of NPRL2 in L-OHP-induced cytotoxicity, NPRL2 was transduced into HCT116 cells. The IC50 of L-OHP was lower in cells transduced with NPRL2 than in NC cells $(\mathrm{P}<0.05)$, indicating that overexpression of NPRL2 markedly increases the sensitivity of HCT116 cells to L-OHP (Fig. 2A). Cell survival was assayed following NPRL2 transduction and treatment with $10 \mu \mathrm{g} / \mathrm{ml} \mathrm{L}-\mathrm{OHP}$ for an additional 24, 48 and $72 \mathrm{~h}$, which confirmed that the effects of NPRL2 on L-OHP sensitivity were time dependent (Fig. 2B). 
A

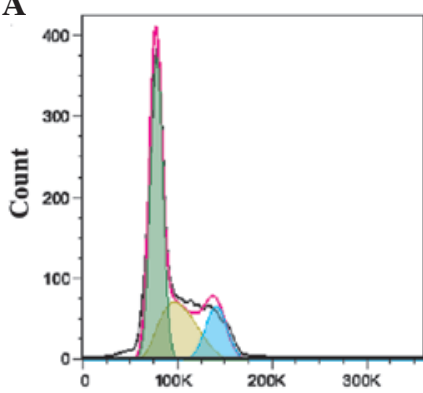

FL2-A:: FL2-A

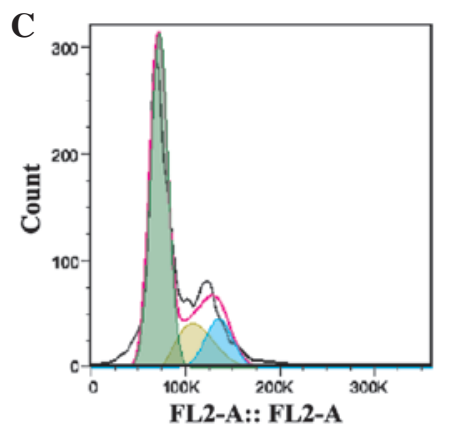

B
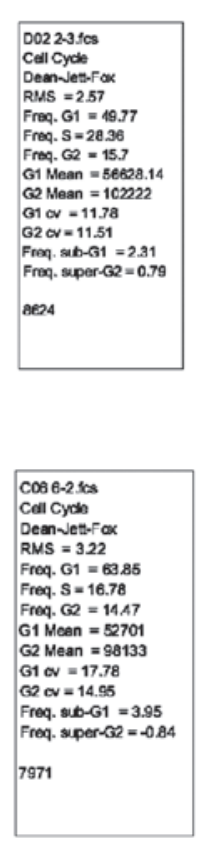

D
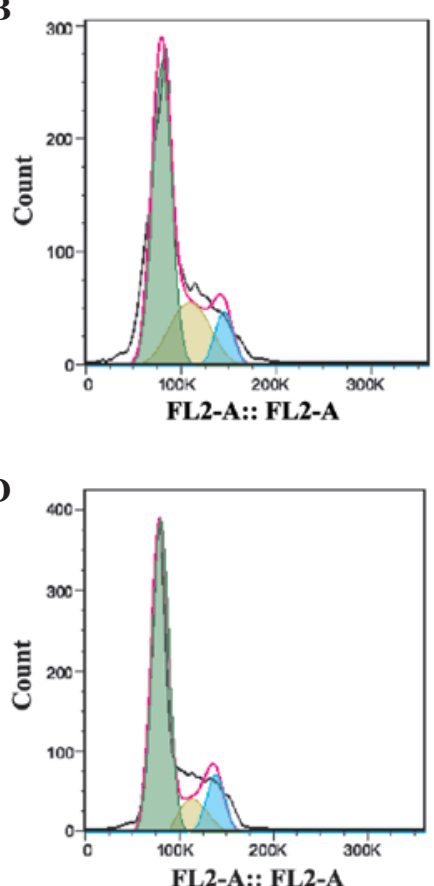
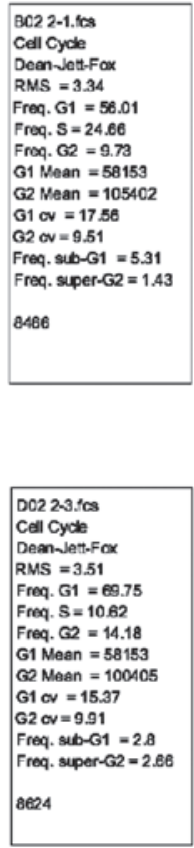

Figure 3. NPRL2 overexpression increases L-OHP sensitivity by inhibiting HCT116 cell growth. (A) NC cells; (B) NPRL2-transduced cells; (C) HCT116 cells treated with $10 \mu \mathrm{g} / \mathrm{ml} \mathrm{L-OHP} \mathrm{and} \mathrm{grown} \mathrm{for} 48 \mathrm{~h}$; (D) NPRL2-transduced HCT116 cells treated with $10 \mu \mathrm{g} / \mathrm{ml} \mathrm{L-OHP}$ and grown for $48 \mathrm{~h}$. NPRL2, nitrogen permease regulator-like 2; L-OHP, oxaliplatin; NC, negative control.

A $\quad F 01$

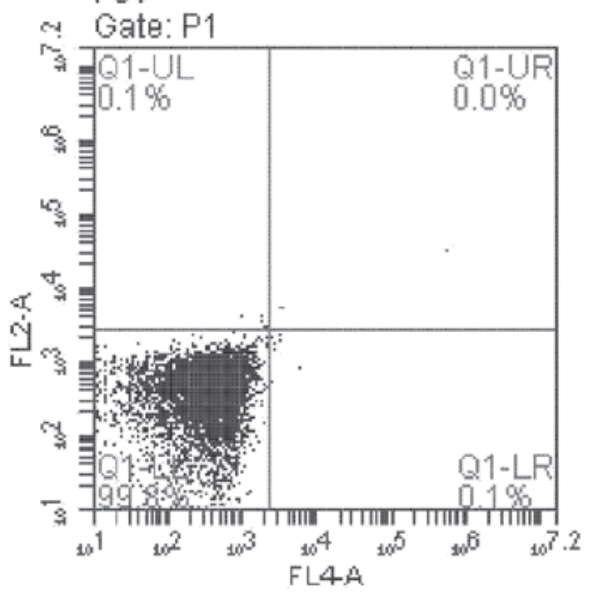

B04

C

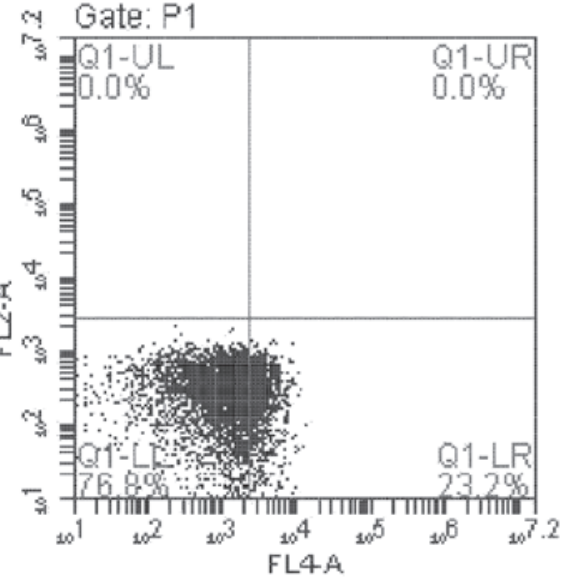

B $\quad F 08$

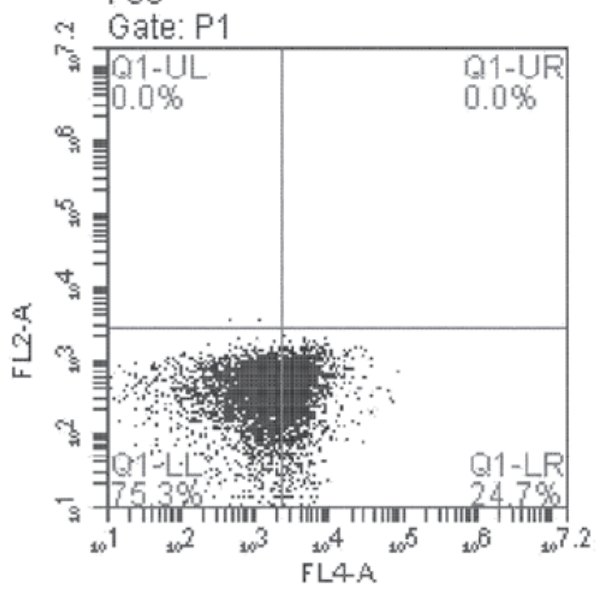

$\mathrm{B} 05$

D

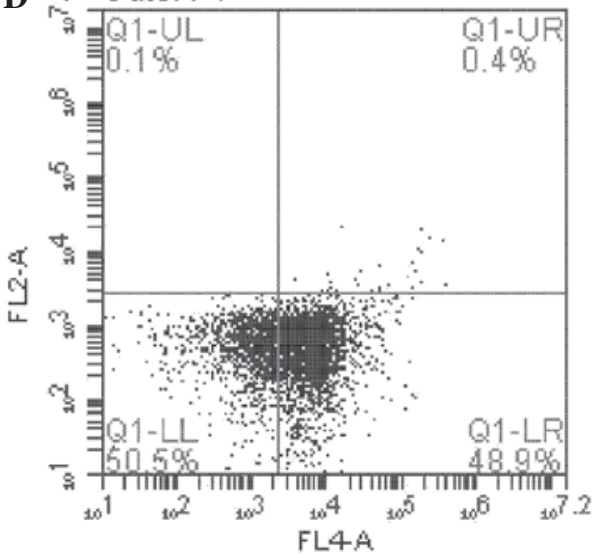

Figure 4. NPRL2 overexpression increases L-OHP sensitivity by promoting apoptosis in HCT116 cells. (A) NC cells; (B) NPRL2-transduced cells; (C) HCT116 cells treated with $10 \mu \mathrm{g} / \mathrm{ml} \mathrm{L-OHP}$ and grown for $48 \mathrm{~h}$; (D) NPRL2-transduced HCT116 cells treated with $10 \mu \mathrm{g} / \mathrm{ml}$ L-OHP and grown for $48 \mathrm{~h}$. NPRL2, nitrogen permease regulator-like 2; L-OHP, oxaliplatin; $\mathrm{NC}$, negative control. 
A $\quad$ B11

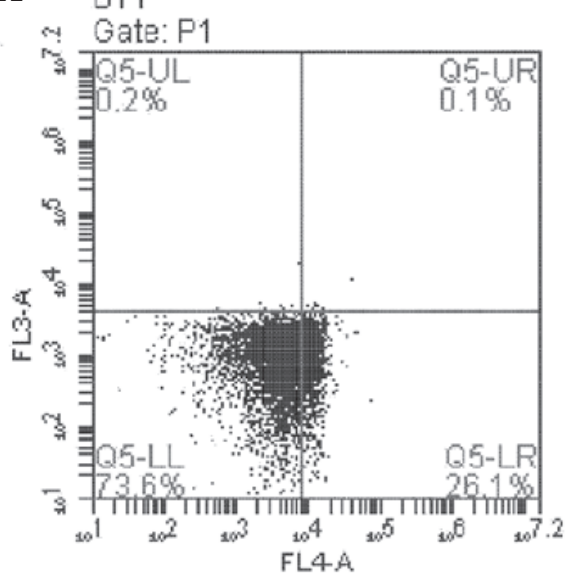

C $\quad$ 11

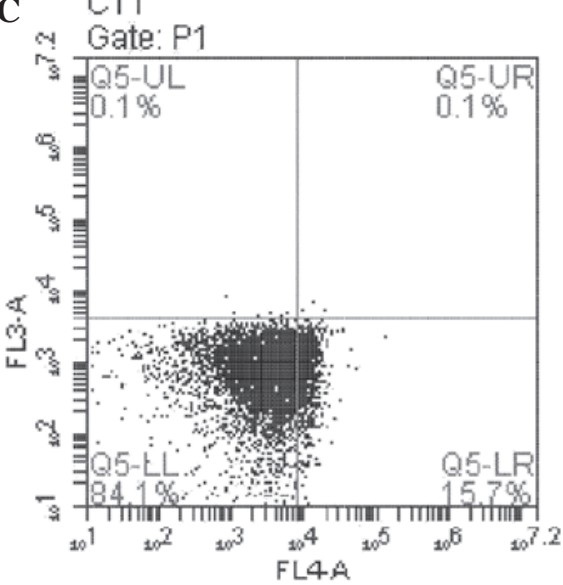

B

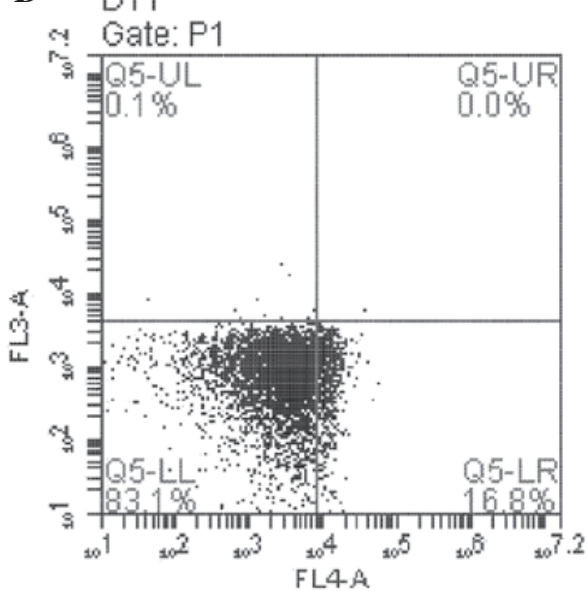

D

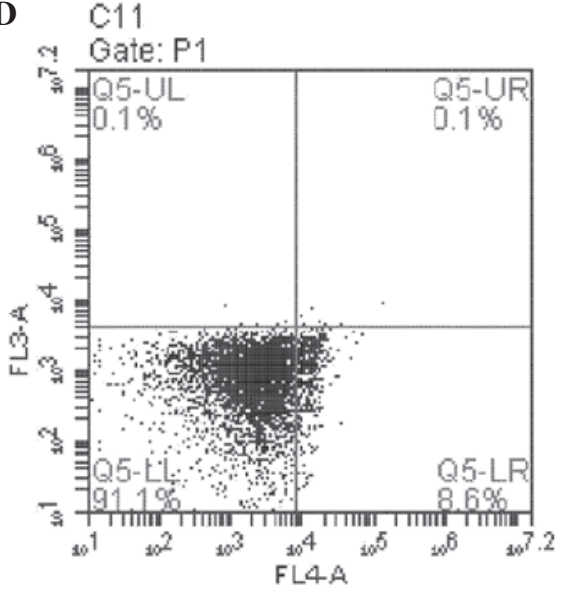

Figure 5. Combined NPRL2 overexpression and L-OHP treatment decreases the rate of CD24+ apoptotic HCT116 cells. (A) NC cells; (B) NPRL2-transduced cells; (C) HCT116 cells treated with $10 \mu \mathrm{g} / \mathrm{ml} \mathrm{L-OHP} \mathrm{and} \mathrm{grown} \mathrm{for} 48 \mathrm{~h}$; (D) NPRL2-transduced HCT116 cells treated with $10 \mu \mathrm{g} / \mathrm{ml} \mathrm{L-OHP} \mathrm{and} \mathrm{grown} \mathrm{for}$ $48 \mathrm{~h}$. NPRL2, nitrogen permease regulator-like 2; L-OHP, oxaliplatin; NC, negative control.

NPRL2 overexpression increases L-OHP sensitivity by inhibiting cell growth. Cell survival assays revealed that the cell cycle was arrested in the G1 phase and that there was a partial decrease in the $S$ phase population following NPRL2 transduction in HCT116 cells $(\mathrm{P}<0.05)$. In addition, L-OHP significantly inhibited the growth of NPRL2-transduced cells compared with NC cells ( $\mathrm{P}<0.01$; Fig. 3$)$.

NPRL2 overexpression increases L-OHP sensitivity by promoting apoptosis. Flow cytometry was performed to investigate the role of NPRL2 in L-OHP-induced apoptosis. The number of apoptotic cells was greater in NPRL2-transduced and L-OHP cells than in NC cells $(\mathrm{P}<0.05)$. In addition, the combination of NPRL2 overexpression and L-OHP treatment promoted apoptosis more significantly than either perturbation alone ( $\mathrm{P}<0.01$; Fig. 4). NPRL2 and L-OHP also decreased the rate of CD24-positive apoptotic HCT116 cells $(\mathrm{P}<0.05)$. In addition, L-OHP significantly decreased the proportion of CD24-positive apoptotic HCT116 cells among cells overexpressing NPRL2 compared with NC cells ( $\mathrm{P}<0.01$; Fig. 5).

NPRL2 promotes HCT116 cell sensitivity to L-OHP by inhibiting the PI3K/Akt/mTOR signaling pathway. The protein expression in the following four different groups of cells was evaluated by western blot analysis: Negative control cells, NPRL2-transduced cells, HCT116 cells treated with $10 \mu \mathrm{g} / \mathrm{ml} \mathrm{L}-\mathrm{OHP}$ and grown for $48 \mathrm{~h}$ and NPRL2-transduced HCT116 cells treated with $10 \mu \mathrm{g} / \mathrm{ml} \mathrm{L-OHP}$ and grown for 48 h. Overexpression of NPRL2 and L-OHP treatment downregulated PDK1, 4E-BP1, phosphorylated PI3K, Akt, mTOR and p70S6K in HCT116 cells. In addition, the combination of NPRL2 overexpression and L-OHP treatment resulted in significantly greater downregulation of these genes compared with either perturbation alone $(\mathrm{P}<0.01)$. Furthermore, L-OHP upregulated caspase-3 and caspase- 9 to promote apoptosis in NPRL2-overexpressing cells compared with cells subjected to either perturbation alone or NC cells $(\mathrm{P}<0.01$; Fig. 6).

\section{Discussion}

CRC is the third leading cause of cancer-associated mortality and the second overall cause in males and females combined in the USA (9). A deep understanding of the dietary, lifestyle and medical risk factors for this malignancy has been achieved $(10,11)$. In China, with the improvement of living standards and alterations in diet, the incidence of CRC has gradually increased. However, half of CRC treatment remains unsuccessful.

For patients with advanced colonic cancer, surgery and systemic chemotherapy are the most common treatment 
A

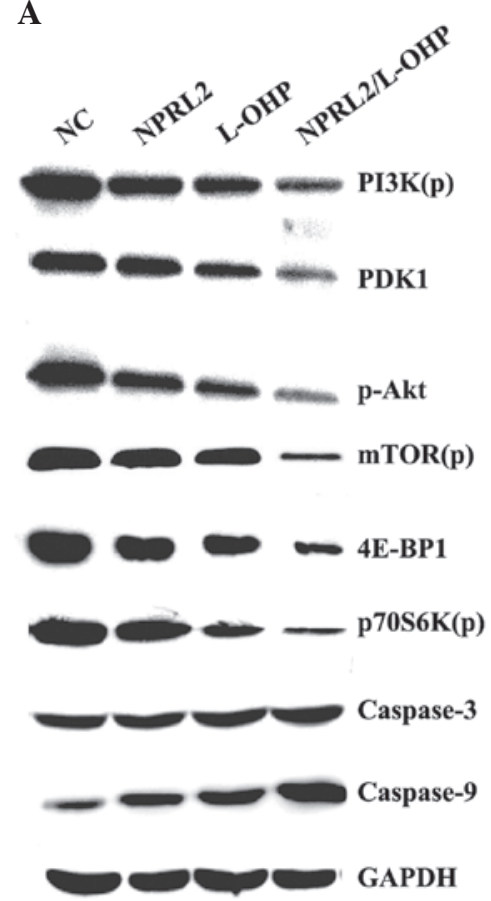

B

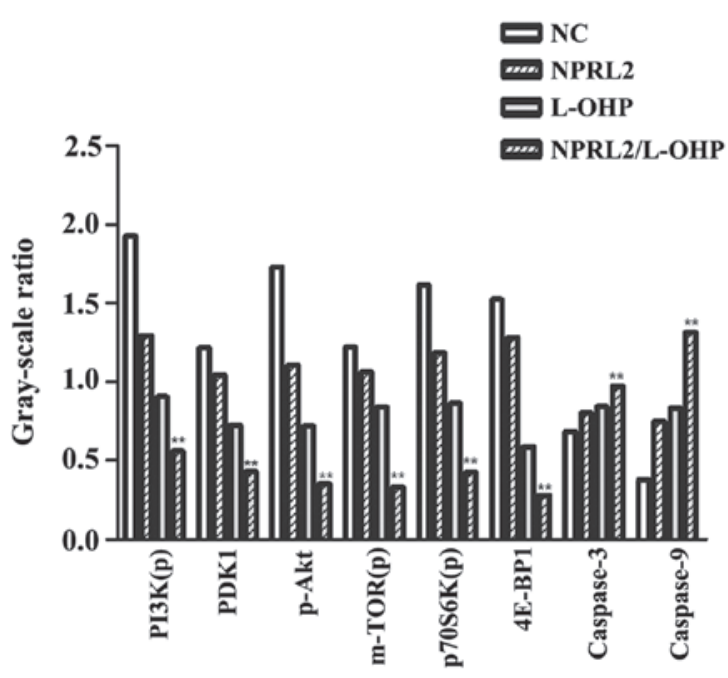

Figure 6. Western blot analysis of the protein levels of PI3K(p), PDK1, p-Akt, mTOR(p), p70S6K(p), 4E-BP1, caspase-3, caspase-9 and GAPDH. (A) Protein expression levels in the four different groups of cells (NC cells; NPRL2-transduced cells; HCT116 cells treated with $10 \mu \mathrm{g} / \mathrm{ml} \mathrm{L}-\mathrm{OHP}$ and grown for $48 \mathrm{~h}$; NPRL2-transduced HCT116 cells treated with $10 \mu \mathrm{g} / \mathrm{ml} \mathrm{L-OHP} \mathrm{and} \mathrm{grown} \mathrm{for} 48 \mathrm{~h}$ ). (B) Gray-scale ratio comparison between the four different groups of cells (NC cells; NPRL2-transduced cells; HCT116 cells treated with $10 \mu \mathrm{g} / \mathrm{ml}$ L-OHP and grown for $48 \mathrm{~h}$; NPRL2-transduced HCT116 cells treated with $10 \mu \mathrm{g} / \mathrm{ml} \mathrm{L}-\mathrm{OHP}$ and grown for $48 \mathrm{~h}$ ). The downregulation of the expression of PDK1, 4E-BP1, phosphorylated PI3K, Akt, mTOR and p70S6K was observed in cells overexpressing NPRL2 and treated with L-OHP compared with NC cells, NPRL2-transduced cells and cells treated with $10 \mu \mathrm{g} / \mathrm{ml} \mathrm{L}-\mathrm{OHP}$. NPRL2 overexpression resulted in increased levels of caspase-3 and caspase-9 compared with NC cells. In addition, combined NPRL2 overexpression and L-OHP treatment significantly upegulated apoptosis compared with either perturbation alone. Western blot analysis was performed using GAPDH as a loading control. ${ }^{* *} \mathrm{P}<0.01$, compared with NC cells, NPRL2-transduced cells, HCT116 cells treated with $10 \mu \mathrm{g} / \mathrm{ml} \mathrm{L-OHP}$ and grown for $48 \mathrm{~h}$. NPRL2, nitrogen permease regulator-like 2; L-OHP, oxaliplatin; NC, negative control; PI3K, phosphatidylinositol 3-kinase; PDK1, pyruvate dehydrogenase kinase, isozyme 1; mTOR, mammalian target of rapamycin; 4E-BP1, 4E-binding protein 1.

methods. L-OHP is an alkylating agent that is cell cycle non-specific and most active during the resting phase of the cell cycle. This drug forms a coordination metal salt complex and inhibits DNA synthesis in cancer cells. Although L-OHP is widely used for the treatment of advanced malignancies, long-term treatment outcomes are unsatisfactory. Cancer recurrence is frequently observed in patients who have undergone chemotherapy and recurrent cancers are frequently highly malignant and drug resistant. Furthermore, tumor chemotherapy is often associated with side effects, complicating the limitation of the long-term effects of chemotherapy (12-17). In addition, tumor chemoresistance can develop as a result of decreased drug uptake, increased drug efflux, activation of detoxifying systems or DNA repair mechanisms and/or evasion of drug-induced apoptosis. Improving the sensitivity of chemotherapy and overcoming L-OHP resistance are critical requirements in cancer therapy.

The NPRL2 gene has potent tumor suppressive activity in vitro and in vivo and has been suggested to be involved in DNA mismatch repair, cell cycle checkpoint signaling and regulation of the apoptotic pathway $(5,18)$. Overexpression of NPRL2 inhibits proliferation and induces apoptosis in a variety of tumor cell lines (19). In the present study, it was demonstrated that NPRL2 overexpression increases L-OHP sensitivity in HCT116 cells. Initially, it was observed that the IC50 of L-OHP was lower in cells transduced with NPRL2 than in $\mathrm{NC}$ cells $(\mathrm{P}<0.05)$. The present study determined that the effects of NPRL2 on L-OHP sensitivity were time dependent and that the overexpression of NPRL2 promotes apoptosis in a time-dependent manner, thereby inhibiting cell proliferation. Following NPRL2 transduction in HCT116 cells, the cell cycle was arrested in the G1 phase and there was a partial decrease in cells in the $\mathrm{S}$ phase $(\mathrm{P}<0.05)$. In addition, $\mathrm{L}-\mathrm{OHP}$ significantly inhibited cell growth in NPRL2-transduced cells compared with $\mathrm{NC}$ cells $(\mathrm{P}<0.01)$. These data further confirm that NPRL 2 overexpression increases L-OHP sensitivity by inhibiting cell growth. Flow cytometric analysis revealed an increase in apoptotic cells due to NPRL2 transduction and L-OHP treatment compared with $\mathrm{NC}$ cells $(\mathrm{P}<0.05)$. In addition, combined NPRL2 overexpression and L-OHP treatment promoted apoptosis more significantly than either perturbation alone. These results indicate that an essential function of exogenous NPRL2 involves activation of the DNA damage checkpoint pathway, which regulates not only cell-cycle checkpoints but also DNA repair, genome maintenance, senescence and apoptosis (20).

NPRL2 may enhance L-OHP sensitivity via additional mechanisms, which require examination in future studies. In the present study, it was demonstrated that NPRL2 is a new potential therapeutic molecule. Compared with traditional drugs, NPRL2 has a more modulatory role. Combined NPRL2 overexpression and L-OHP treatment effectively 
downregulated the phosphorylation of PI3K, Akt and mTOR and the mTOR downstream target proteins phospho-p70S6K (Thr389) and 4E-BP1 (Thr37/46). The PI3K/Akt/mTOR signaling axis is critical in proliferation, apoptotic resistance, angiogenesis and metastasis and is central to the development and maintenance of CRC (21). Previous studies have reported the potential for the PI3K/Akt/mTOR network to be therapeutically targeted at multiple molecular levels $(22,23)$. PI3K is activated upon the binding of growth factors to their cognate receptors. Activated PI3K leads to Akt activation via phosphorylation at Ser473 and Thr308 (24). Akt activates several downstream targets, including mTOR. Deregulation of mTOR signaling occurs in several types of human tumor, including colon cancer (21). mTOR associates with Raptor (mTORC1 complex) to phosphorylate p70S6K, which in turn phosphorylates 4E-BP1, leading to increased cell proliferation (25). 4E-BP1 is considered to be a funneling factor through which transforming signals converge, channeling oncogenic proliferative signals regardless of the specific upstream oncogenic alteration (26). Phospho-p70S6K is cytoplasmic and its nuclear immunopositivity is a common feature of various types of tumor. Phospho-p70S6K stimulates ribosome rearrangement into active polysomes and increases the capacity of the translational events essential for the G1/S transition of the cell cycle (27). These findings suggest that NPRL2 overexpression enhances L-OHP sensitivity by downregulating the functions of the PI3K/Akt/mTOR network, leading to inhibition of cell proliferation and G1 cell cycle arrest. Furthermore, L-OHP upregulates caspase-3 and caspase-9 to promote apoptosis in NPRL2-overexpressing cells compared with either perturbation alone and NC cells $(\mathrm{P}<0.01)$. Furthermore, the present study demonstrated that the NPRL2-mediated increase in L-OHP sensitivity that induces apoptosis in HCT116 cells is associated with significant activation of caspase-3 and caspase-9.

Notably, a previous study also demonstrated that combined NPRL2 transduction and L-OHP treatment led to a significant decrease in the proportion of $\mathrm{CD} 24^{+}$apoptotic HCT116 cells, indicating that NPRL2 overexpression causes downregulation of the proportion of $\mathrm{CD} 24^{+}$cells. CD24 is a sialoglycoprotein that is anchored to the cell surface by a glycosyl phosphatidylinositol linkage (28). This protein is a ligand for P-selectin, an adhesion receptor found on activated endothelial cells and platelets; thus, it may contribute to the metastasizing capacity of CD24-expressing tumor cells $(29,30)$. In gastric cancer, an association between high CD24 expression and lymph node metastasis, venous invasion and lymphatic invasion has been observed (31). CD24 expression tended to be higher in cell lines derived from differentiated gastric carcinomas, including those derived from lymph node metastases (32). Downregulation of CD24 by NPRL 2 overexpression may significantly reduce tumor invasiveness and the metastatic capacity of HCT116 cells.

In conclusion, transfection of colon cancer cells with NPRL2 resulted in a significant inhibition of tumor cell growth. The present study also demonstrated that NPRL2 affects the PI3K/Akt/mTOR pathway. It was confirmed that NPRL2 enhances L-OHP sensitivity by inhibiting proliferation and promoting apoptosis and may potentially serve as a therapeutic target for overcoming L-OHP resistance in colon cancer. These mechanisms are likely active in other types of cancer and may be exploited for the development of novel cancer therapies.

\section{References}

1. Perazzo F, Piaggio F, Krupitzki H, et al: Clinical-pathological features and gene profile in colorectal cancer. Medicina (B Aires) 73: 417-422, 2013 (In Spanish).

2. Ahmed FE: Gene-gene, gene-environment \& multiple interactions in colorectal cancer. J Environ Sci Health C Environ Carcinog Ecotoxicol Rev 24: 1-101, 2006.

3. Patel BB and Majumdar AP: Synergistic role of curcumin with current therapeutics in colorectal cancer: minireview. Nutr Cancer 61: 842-846, 2009.

4. Lerman MI and Minna JD: The 630-kb lung cancer homozygous deletion region on human chromosome 3p21.3: identification and evaluation of the resident candidate tumor suppressor genes. Cancer Res 60: 6116-6133, 2000.

5. Wistuba II, Behrens C, Virmani AK, Mele G, et al: High resolution chromosome $3 \mathrm{p}$ allelotyping of human lung cancer and preneoplastic/preinvasive bronchial epithelium reveals multiple, discontinuous sites of $3 p$ allele loss and three regions of frequent breakpoints. Cancer Res 60: 1949-1960, 2000.

6. Li J, Wang F, Haraldson K, Protopopov A, et al: Functional characterization of the candidate tumor suppressor gene NPRL2/G21 located in 3p21.3C. Cancer Res 64: 6438-6443, 2004.

7. Schenk PW, Brok M, Boersma AW, et al: Anticancer drug resistance induced by disruption of the Saccharomyces cerevisiae NPR2 gene: a novel component involved in cisplatin- and doxorubicin-provoked cell kill. Mol Pharmacol 64: 259-268, 2003.

8. Ueda K, Kawashima H, Ohtani S, et al: The 3p21.3 tumor suppressor NPRL2 plays an important role in cisplatin-induced resistance in human non-small-cell lung cancer cells. Cancer Res 66: 9682-9690, 2006.

9. Chan AT and Giovannucci EL: Primary prevention of colorectal cancer. Gastroenterology 138: 2029-2043, 2010.

10. Poynter JN, Haile RW, Siegmund KD, et al: Colon Cancer Family Registry: Associations between smoking, alcohol consumption and colorectal cancer, overall and by tumor microsatellite instability status. Cancer Epidemiol Biomarkers Prev 18: 2745-2750, 2009.

11. Bostick RM, Potter JD, Kushi LH, Sellers TA, Steinmetz KA, McKenzie DR, Gapstur SM and Folsom AR: Sugar, meat and fat intake and non-dietary risk factors for colon cancer incidence in Iowa women (United States). Cancer Causes Control 5: 38-52, 1994.

12. Yang AD, Fan F, Camp ER, van Buren G, Liu W, et al: Chronic oxaliplatin resistance induces epithelial-to-mesenchymal transition in colorectal cancer cell lines. Clin Cancer Res 12: 4147-4153, 2006.

13. Shah AN, Summy JM, Zhang J, Park SI, Parikh NU, et al: Development and characterization of gemcitabine-resistant pancreatic tumor cells. Ann Surg Oncol 14: 3629-3637, 2007.

14. De Larco JE, Wuertz BR, Manivel JC and Furcht LT: Progression and enhancement of metastatic potential after exposure of tumor cells to chemotherapeutic agents. Cancer Res 61: 2857-2861, 2001.

15. Kajiyama H, Shibata K, Terauchi M, Yamashita M, Ino K, et al: Chemoresistance to paclitaxel induces epithelial-mesenchymal transition and enhances metastatic potential for epithelial ovarian carcinoma cells. Int J Oncol 31: 277-283, 2007.

16. Xiong W, Ren ZG, Qiu SJ, Sun HC, Wang L, et al: Residual hepatocellular carcinoma after oxaliplatin treatment has increased metastatic potential in a nude mouse model and is attenuated by Songyou Yin. BMC Cancer 10: 219, 2010.

17. Yamauchi K, Yang M, Hayashi K, Jiang P, Yamamoto N, et al: Induction of cancer metastasis by cyclophosphamide pretreatment of host mice: an opposite effect of chemotherapy. Cancer Res 68: 516-520, 2008

18. Zabarovsky ER, Lerman MI and Minna JD: Tumor suppressor genes on chromosome $3 p$ involved in the pathogenesis of lung and other cancers. Oncogene 21: 6915-6935, 2002.

19. Ji L, Nishizaki M, Gao B, et al: Expression of several genes in the human chromosome 3p21.3 homozygous deletion region by an adenovirus vector results in tumor suppressor activities in vitro and in vivo. Cancer Res 62: 2715-2720, 2002. 
20. Zhou BB and Elledge SJ: The DNA damage response: putting checkpoints in perspective. Nature 408: 433-439, 2000.

21. Johnson SM, Gulhati P, Rampy BA, Han Y, Rychahou PG, Doan HQ, et al: Novel expression patterns of PI3K/Akt/mTOR signaling pathway components in colorectal cancer. J Am Coll Surg 210: 767-776, 2010.

22. Maira SM, Voliva C and Garcia-Echeverria C: Class IA phosphatidylinositol 3-kinase: from their biologic implication in human cancers to drug discovery. Expert Opin Ther Targets 12: 223-238, 2008.

23. Ekstrand AI, Jonsson M, Lindblom A, Borg A and Nilbert M: Frequent alterations of the PI3K/AKT/mTOR pathways in hereditary nonpolyposis colorectal cancer. Fam Cancer 9 : 125-129, 2010.

24. Awasthi N, Yen PL, Schwarz MA and Schwarz RE: The efficacy of a novel, dual PI3K/mTOR inhibitor NVP-BEZ235 to enhance chemotherapy and antiangiogenic response in pancreatic cancer. J Cell Biochem 113: 784-791, 2012.

25. Glienke W, Maute L, Wicht J and Bergmann L: The dual PI3K/mTOR inhibitor NVP-BGT226 induces cell cycle arrest and regulates Survivin gene expression in human pancreatic cancer cell lines. Tumour Biol 33: 757-765, 2012.
26. Armegnol G, Rojo F, Castellví J, et al: 4E-binding protein 1: a key molecular 'funnel factor' in human cancer with clinical implications. Cancer Res 67: 7551-7555, 2007.

27. Xu G, Zhang W, Bertram P, et al: Pharmacogenomic profiling of the PI3K/PTEN-AKT-mTOR pathway in common human tumors. Int J Oncol 24: 893-900, 2004.

28. Lim SC and Oh SH: The role of CD24 in various human epithelial neoplasias. Pathol Res Pract 201: 479-486, 2005.

29. Sammar M, Aigner S, Hubbe M, Schirrmacher V, Schachner M, Vestweber D and Altevogt P: Heat-stable antigen (CD24) as ligand for mouse P-selectin. Int Immunol 6: 1027-1036, 1994.

30. Aigner S, Ramos CL, Hafezi-Moghadam A, Lawrence MB, Friederichs J, Altevogt P and Ley K: CD24 mediates rolling of breast carcinoma cells on P-selectin. FASEB J 12: 1241-1251, 1998.

31. Yong CS, Ou Yang CM, Chou YH, Liao CS, Lee CW and Lee CC: CD44/CD24 expression in recurrent gastric cancer: A retrospective analysis. BMC Gastroenterol 12: 95, 2012.

32. Takahashi M, Nakajima M, Ogata H, Domeki Y, Ohtsuka K, Ihara K, Kurayama E, Yamaguchi S, Sasaki K, Miyachi K and Kato H: CD24 expression is associated with progression of gastric cancer. Hepatogastroenterology 60: 653-658, 2013. 